\title{
Chapter 91 \\ Report on the Global Environment \\ Competitiveness of Mauritania
}

Mauritania is located in West Africa. It covers 1,030,700 square kilometres and is bordered by Atlantic Ocean in the west, by Western Sahara in the north, by Algeria in the northeast, by Mali in the east and southeast, and by Senegal in the southwest. It is located in tropical desert climate. It had a population of 3.54 million and domestic

Score: 39.27

Rank: 129 production the gross (GDP) of USD 4.08 billion in 2011. Through the evaluation of global environment competitiveness Mauritania, we can know that the environment competitiveness index of Slovenia ranks at 129 among 133 countries.

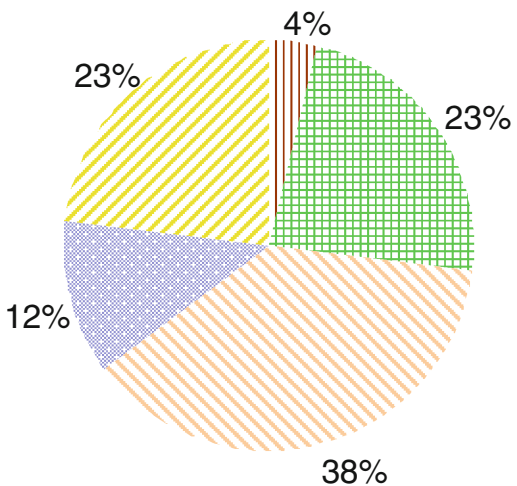

II Resource Environment Competitiveness

* Ecological Environment Competitiveness

Environment Carrying Competitiveness

- Environment Management Competitiveness

Fig. 91.1 Contribution of Environment Harmony sub-index of GEC

Competitiveness 


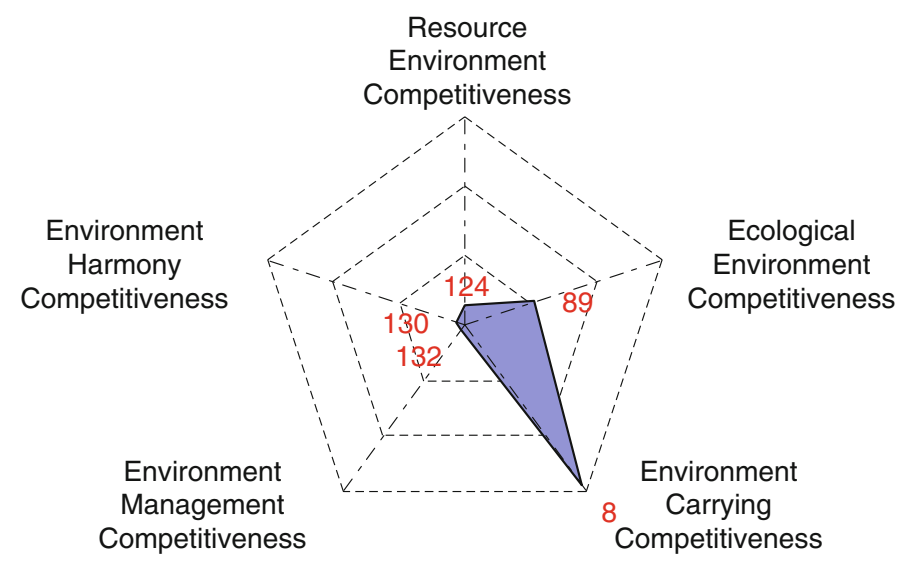

Fig. 91.2 Rank of sub-index of GEC

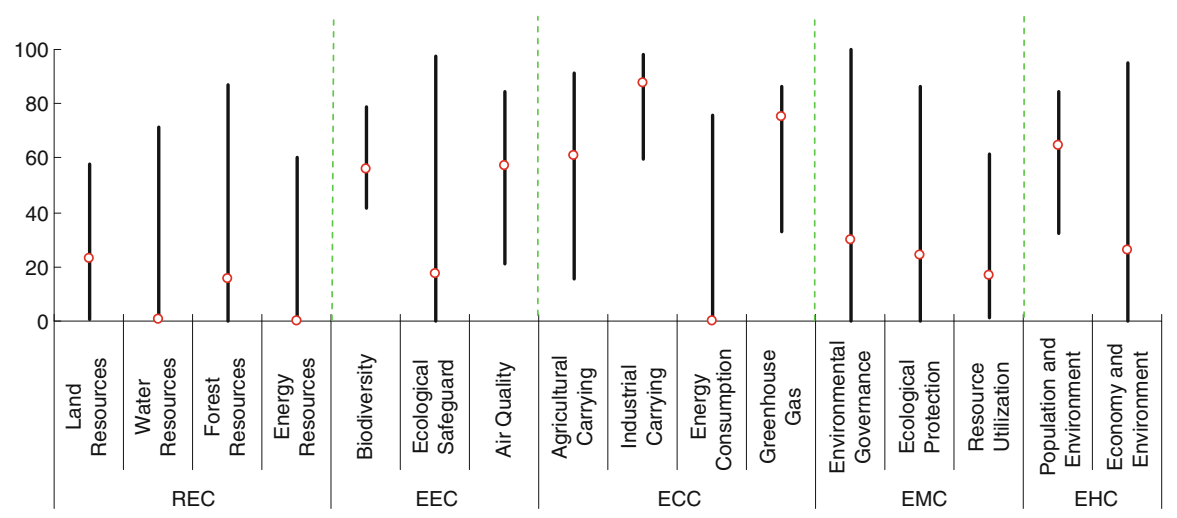

Fig. 91.3 Score and rank of the pillars of GEC

Table 91.1 Score and rank of all indicators of GEC

\begin{tabular}{|c|c|c|c|c|c|}
\hline Indicators & Score & Rank & Indicators & Score & Rank \\
\hline 1 Resource En & 7.88 & 124 & 1.3 Forest Resources & 15.25 & 122 \\
\hline Competitiveness & & & \multirow{2}{*}{$\begin{array}{l}\text { Growing stock in forest } \\
\text { and other wooded land }\end{array}$} & \multirow[t]{2}{*}{50.02} & \multirow{2}{*}{114} \\
\hline 1.1 Land Resources & 22.93 & 24 & & & \\
\hline Land area per capita & 52.44 & 4 & \multirow{2}{*}{$\begin{array}{l}\text { Proportion of land area } \\
\text { covered by forest }\end{array}$} & \multirow[t]{2}{*}{0.27} & \multirow[t]{2}{*}{127} \\
\hline Percentage of arable land & 0.57 & 131 & & & \\
\hline to total land area & & & Forest area per capita & 0.47 & 104 \\
\hline Arable land per capita & 5.94 & 84 & 1.4 Energy Resources & 0.00 & 125 \\
\hline 1.2 Water Resources & 0.81 & 128 & Fossil energy & 0.00 & 64 \\
\hline Surface water & 0.12 & 120 & Energy production & 0.00 & 126 \\
\hline Annual precipitation & 3.12 & 125 & \multirow{3}{*}{$\begin{array}{l}\text { Proportion of combustible } \\
\text { renewables and waste } \\
\text { to total energy } \\
\text { consumption }\end{array}$} & \multirow[t]{3}{*}{ N/A } & \multirow[t]{3}{*}{ N/A } \\
\hline Groundwater & 0.00 & 128 & & & \\
\hline $\begin{array}{l}\text { Total internal renewable } \\
\text { water resources }\end{array}$ & 0.02 & 132 & & & \\
\hline
\end{tabular}


Table 91.1 (continued)

\begin{tabular}{|c|c|c|c|c|c|}
\hline Indicators & Score & Rank & Indicators & Score & Rank \\
\hline $\begin{array}{l}\text { Net energy imports } \\
\text { of the energy }\end{array}$ & N/A & N/A & $\begin{array}{l}\text { Ratio of clean energy } \\
\text { consumption }\end{array}$ & 0.00 & 116 \\
\hline consumption & & & Elasticity of energy & N/A & N/A \\
\hline 2 Ecological Environment & 44.87 & 89 & consumption & & \\
\hline Competitiveness & 5587 & & $\begin{array}{l}\text { Elasticity of electric power } \\
\text { consumption }\end{array}$ & N/A & N/A \\
\hline $\begin{array}{l}2.1 \text { Biodiversity } \\
\text { Threatened fish species }\end{array}$ & $\begin{array}{l}55.81 \\
84.91\end{array}$ & $\begin{array}{r}101 \\
78\end{array}$ & 3.4 Greenhouse Gas & 75.11 & 10 \\
\hline $\begin{array}{l}\text { Threatened mammal } \\
\text { species }\end{array}$ & 91.85 & 84 & $\begin{array}{l}\text { Growth rate of } \mathrm{CO}_{2} \\
\text { emissions }\end{array}$ & 62.67 & 30 \\
\hline Threatened plant species & 100.00 & 1 & Growth rate of Methane & N/A & N/A \\
\hline GEF benefits index for & 1.30 & 78 & emissions & & \\
\hline $\begin{array}{l}\text { biodiversity } \\
\text { 2.2 Ecological Safeguard }\end{array}$ & 17.65 & 76 & $\begin{array}{l}\mathrm{CO}_{2} \text { emissions per unit } \\
\text { of land area }\end{array}$ & 100.00 & 3 \\
\hline Terrestrial protected areas & 1.09 & 124 & $\mathrm{CO}_{2}$ emissions per unit & N/A & N/A \\
\hline Marine protected areas & 42.50 & 6 & of energy consumption & & \\
\hline 2.3 Air Quality & 57.04 & 71 & 4 Environment Management & 23.73 & 132 \\
\hline Inhalable particles (PM10) & 50.36 & 118 & Competitiveness & & \\
\hline Particulate matter (PM2.5) & 74.49 & 98 & 4.1 Environmental & 30.00 & 130 \\
\hline $\begin{array}{l}\text { Index of indoor air } \\
\text { pollution }\end{array}$ & 6.90 & 103 & $\begin{array}{l}\text { Governance } \\
\text { Agricultural chemicals }\end{array}$ & 0.00 & 86 \\
\hline Nitrogen oxides emission & 100.00 & 1 & regulation & & \\
\hline Sulfur dioxide emission & 100.00 & 1 & Percentage of the rural & 48.00 & 109 \\
\hline $\begin{array}{l}3 \text { Environment Carrying } \\
\text { Competitiveness }\end{array}$ & 74.52 & 8 & $\begin{array}{l}\text { population with access } \\
\text { to an improved water } \\
\text { source }\end{array}$ & & \\
\hline 3.1 Agricultural Carrying & 60.96 & 111 & & & 130 \\
\hline $\begin{array}{l}\text { Cereal yield per unit } \\
\text { of arable land }\end{array}$ & 6.47 & 121 & $\begin{array}{l}\text { Percentage of the urban } \\
\text { population with access } \\
\text { to an improved water }\end{array}$ & 52.00 & 130 \\
\hline Fertilizer consumption per & 99.66 & 15 & source & & \\
\hline $\begin{array}{l}\text { unit of arable land } \\
\text { Annual freshwater } \\
\text { withdrawals for }\end{array}$ & 94.91 & 94 & $\begin{array}{l}\text { 4.2 Ecological Protection } \\
\text { Area of plantation and } \\
\text { afforestation }\end{array}$ & $\begin{array}{r}24.43 \\
0.03\end{array}$ & $\begin{array}{r}94 \\
104\end{array}$ \\
\hline $\begin{array}{l}\text { agriculture per unit } \\
\text { of arable land }\end{array}$ & & & Biome protect & 3.20 & 125 \\
\hline 3.2 Industrial Carrying & 87.69 & 97 & $\begin{array}{l}\text { Overfishing of fishing } \\
\text { resources }\end{array}$ & 78.21 & 7 \\
\hline $\begin{array}{l}\text { Net exports as a percentage } \\
\text { of GDP }\end{array}$ & 64.94 & 110 & 4.3 Resource Utilization & 16.51 & 130 \\
\hline Electric power & N/A & N/A & $\begin{array}{l}\text { Utilization rate of water } \\
\text { resources }\end{array}$ & 0.56 & 54 \\
\hline value added of industry & & & Percentage of total internal & 3.51 & 125 \\
\hline $\begin{array}{l}\mathrm{SO}_{2} \text { emissions per unit of } \\
\text { value added of industry }\end{array}$ & 100.00 & 1 & $\begin{array}{l}\text { renewable water } \\
\text { resources to total water } \\
\text { resources }\end{array}$ & & \\
\hline $\begin{array}{l}\text { Annual freshwater } \\
\text { withdrawals for }\end{array}$ & 98.13 & 57 & $\begin{array}{l}\text { Percentage of agricultural } \\
\text { land to total land area }\end{array}$ & 45.47 & 80 \\
\hline $\begin{array}{l}\text { 1ndustry per value } \\
\text { added of industry }\end{array}$ & & & Percentage of fossil fuel & N/A & N/A \\
\hline 3.3 Energy Consumption & N/A & N/A & to total energy & & \\
\hline $\begin{array}{l}\text { Energy consumption per } \\
\text { unit of land area }\end{array}$ & N/A & N/A & & & \\
\hline
\end{tabular}


Table 91.1 (continued)

\begin{tabular}{|c|c|c|c|c|c|}
\hline Indicators & Score & Rank & Indicators & Score & Rank \\
\hline $\begin{array}{l}5 \text { Environment Harmony } \\
\text { Competitiveness }\end{array}$ & 45.37 & 130 & $\begin{array}{l}\mathrm{CO}_{2} \text { emissions (metric tons } \\
\text { per capita) }\end{array}$ & 98.51 & 26 \\
\hline $\begin{array}{l}\text { 5.1 Population and } \\
\text { Environment }\end{array}$ & 64.62 & 101 & $\begin{array}{l}\text { Energy consumption } \\
\text { per capita }\end{array}$ & N/A & N/A \\
\hline $\begin{array}{l}\text { Improved sanitation } \\
\text { facilities }\end{array}$ & 26.00 & 117 & $\begin{array}{l}\text { 5.2 Economy and } \\
\text { Environment }\end{array}$ & 26.13 & 128 \\
\hline $\begin{array}{l}\text { (\% of population } \\
\text { with access) }\end{array}$ & & & $\begin{array}{l}\text { Land resource utilization } \\
\text { efficiency }\end{array}$ & 0.00 & 133 \\
\hline $\begin{array}{l}\text { Motor vehicles } \\
\quad \text { (per 1,000 people) }\end{array}$ & 99.63 & 5 & $\begin{array}{l}\text { Sulfur dioxide } \\
\text { emissions per unit }\end{array}$ & 100.00 & 1 \\
\hline \multirow{2}{*}{$\begin{array}{l}\text { Renewable internal } \\
\text { freshwater resources } \\
\text { per capita }\end{array}$} & \multirow[t]{2}{*}{0.14} & \multirow[t]{2}{*}{124} & of GDP & & \\
\hline & & & $\begin{array}{l}\text { Carbon dioxide emissions } \\
\text { per unit of GDP }\end{array}$ & 79.72 & 92 \\
\hline $\begin{array}{l}\mathrm{SO}_{2} \text { emissions (metric tons } \\
\text { per capita) }\end{array}$ & 100.00 & 1 & $\begin{array}{l}\text { Energy consumption } \\
\text { per unit of GDP }\end{array}$ & N/A & N/A \\
\hline
\end{tabular}

Table 91.2 Rank distribution of the individual indicators of GEC

\begin{tabular}{|c|c|c|c|c|c|c|}
\hline Sub-index & $\begin{array}{l}\text { Number } \\
\text { of the } \\
\text { individual } \\
\text { indicators }\end{array}$ & $\begin{array}{l}\text { Rank } \\
1-10\end{array}$ & $\begin{array}{l}\text { Rank } \\
11-30\end{array}$ & $\begin{array}{l}\text { Rank } \\
31-60\end{array}$ & $\begin{array}{l}\text { Rank } \\
61-100\end{array}$ & $\begin{array}{l}\text { Rank } \\
101-133\end{array}$ \\
\hline $\begin{array}{l}\text { Resource Environment } \\
\text { Competitiveness }\end{array}$ & 14 & 1 & 1 & 0 & 1 & 10 \\
\hline $\begin{array}{l}\text { Ecological Environment } \\
\text { Competitiveness }\end{array}$ & 11 & 2 & 0 & 0 & 5 & 4 \\
\hline $\begin{array}{l}\text { Environment Carrying } \\
\text { Competitiveness }\end{array}$ & 15 & 3 & 2 & 0 & 1 & 4 \\
\hline $\begin{array}{l}\text { Environment Management } \\
\text { Competitiveness }\end{array}$ & 10 & 0 & 0 & 1 & 3 & 6 \\
\hline $\begin{array}{l}\text { Environment Harmony } \\
\text { Competitiveness }\end{array}$ & 10 & 3 & 1 & 0 & 1 & 5 \\
\hline Total & 60 & 9 & 4 & 1 & 11 & 29 \\
\hline
\end{tabular}

Open Access This chapter is distributed under the terms of the Creative Commons Attribution Noncommercial License, which permits any noncommercial use, distribution, and reproduction in any medium, provided the original author(s) and source are credited. 